\title{
LIX. The invisible solar and lunar spectrum
}

\section{S.P. Langley}

To cite this article: S.P. Langley (1888) LIX. The invisible solar and lunar spectrum, Philosophical Magazine Series 5, 26:163, 505-520, DOI: $10.1080 / 14786448808628306$

To link to this article: http://dx.doi.org/10.1080/14786448808628306

曲 Published online: 29 Apr 2009.

6 Submit your article to this journal $\pi$

Џ Article views: 2

Q View related articles $₫$ 
a slight visible discharge from the edge of the foil on the back of the plate, and probably therefore the same from that on the film to which the edge-marking may be due. There was also on the plate much blotchy marking, apparently corresponding to the wrinkling of the foil in contact with the film.

With a piece of gutta-percha tissue between the stencilplate foil and the film, the result was similar, only the line round the edges was narrower and the blotching less marked, hence the initials came out more distinctly. When four thicknesses of gutta-percha were interposed, there appeared only blotchy markings on the part under the foil.

These results would go to show that actual disruptive discharge over or in the film is not needed to produce an effect visible on development, but that the figures are produced partly at least by direct electric action on the sensitive film without the intervention of a visibly Iuminous action, or what would be usually understood as a purely photo-chemical cause. Possibly further investigation may show that we have here a new kind of experimental evidence on the relation of electricity to light.

I may add that it is necessary, especially in the experiments with the terminals on the back of the plate, to use rather sensitive plates ; "60 times" plates do very well, while slow plates give imperfect figures in all cases and show almost nothing with the terminals on the back.

\section{The Invisible Solar and Lunar Spectrum. By S. P. LangleY*.}

THEE following investigation has been made from studies at the Allegheny Observatory ; but it is proper to state that the provision of the very special apparatus used is due to the liberality of a citizen of Pittsburgh, who has desired that his name should not be mentioned.

This paper is an abstract of a forthcoming memoir, which will eventually appear in the fourth volume of the publications of the United States National Academy of Sciences, to which the reader is referred for fuller details.

* Communicated by the Author, to whom we are liliewise indebted for the clichés.

As the writer has already presented to the National Academy a memoir (read October 17, 1884, Memoirs Nat. Acad. of Sci., vol. iii.) on the heat of the moon, in which he spoke of investigations still in progress on it, it should be said that these are not yet published, and that they are only given here so far as is necessary in explanation of certain anomalies in the infra-red solar-heat spectrum, which forms the principal subject of the present paper. 
Ever since the writer first* investigated the infra-red of the solar spectrum to the extent of about three microns, he has assumed, from all analogy, the probable existence of solar heat of still greater wave-lengths, which, however, he has not till lately been able to experimentally demonstrate; so that there has been a doubt whether such waves were emitted by the sun after absorption by its own atmosphere, or whether they existed previously to absorption by the earth's atmosphere, and, owing to the action of the latter, never reached us. Below the point $2^{\mu} \cdot 8$, to which the maps published in 1882 and 1883 extended, it was stated, however, at that time that there had apparently been detected feeble, or, more properly speaking, dubious, indications of solar energy. This doubt arose partly from this extreme feebleness here of the heat itself, partly from lack of the usual experimental means, since the glass of our prisms (which, as we had discovered, transmitted the greater part of all the sun's invisible heat then known) absorbed this, while no maker could then supply its place with suitable rock-salt; and, most of all, from a difficulty of a less familiar kind, but which should from its importance be clearly apprehended by the reader. This is, that even if we could recognize that some feeble invisible heat existed, there were then no means of determining that it really belonged to the part of the spectrum where it was found, and was not intruded invisible heat of a more refrangible kind, diffused from its proper place in the upper spectrum by the inevitable action of the spectroscopic apparatus.

In 1884 and 1885, while investigating the invisible spectrum of the sunlit side of the moon, we first found evidence of heat in this region from any extra-terrestrial source-heat whose enormous wave-length was comparable to that chiefly radiated from ice, which was also experimented on. This was so far distinct from the reflected solar heat of greater refrangibility, which occupied its own part of the spectrum, that our experiments indicated that it was chiefly not reflected, but radiated from a surface at a low temperature. But the chief anomaly was that, while we had thus definitely recognized this kind of heat in the extremely feeble heat-spectrum of the moon, we had not yet done so in the far stronger solar one, or, as I observed at the time, that "wo here seem to have heat from the moon of lower wave-length than from the sun."

I do not state (it must be observed) that the sun's heat here is less than the moon's, but that what there is is harder to

* Comptes Rendus de l'Institut de France, September 11, 1882. Amer. Journ. of Science, March 1883; Phil. Mag. March 1883. 
recognize. It is not easy to give an adequate idea of the difficulties of observation which lead to this apparently paradoxical result, particularly as physicists are so far from having yet investigated this region that even the barriers which have closed it to research are themselves of an unfamiliar kind. I can perhaps best illustrate it by analogy. Every spectroscopist knows how very hard it is to view the lines below $\mathrm{A}$; and that even A itself, though very large, is not an easy object to see without special precautions. This arises not so much from the fact that the very deep red light here, like that of dull glowing iron, feebly affects the eye, but, in a still greater degree, because yellow and orange light exists in relatively enormous quantity in the neighbouring parts of the visible spectrum, and that irregularly diffused and reflected portions of this light reappear where they do not belong and overpower the radiation legitimately there. Still we can put a coloured glass before the slit and cut off the intruding light in a great measure, and we can see the extraneous light which comes in, and allow in some degree for its effects ; but here, in the actual case of the unseen heat in the far more remote spectral region we are about to describe, all radiations, both the feeble ones we would study, and the intruders on them which we would avoid, are alike invisible, and we are, of course, unable in any case to use glass, since this is opaque to all the rays now in question. If any one familiar with the visible spectrum will imagine himself as trying to discriminate with his eyes shut between these different components of the apparent radiation just below Fraunhofer's $A$, and endeavouring while blindfold to say how much of it legitimately belongs there and how much does not, he will have a better conception of the difficulties peculiar to our actual field of research, though still an inadequate one, since the total heat radiation here is at best less than the hundredth part of that in the vicinity of the A line, which we have used in illustration.

For the clearer understanding of this I must, in anticipation of what follows, remark that while in the solar spectrum the maximum heat, as we all know, appears not very far from the red, so that the heat corresponding in a general sense to the short waves is great, and to still longer ones small, in the lunar invisible spectrum the reverse is the case; for here, speaking generally, the solar reflected heat found in the upper part of the lunar spectrum is less than the heat apparently radiated from the moon's own soil, which is of great wavelength, and which we have found in the extreme region of the spectrum we are now studying. In other words, the typical solar-spectrum heat is greatest in the relatively short wave- 
lengths; the typical lunar-spectrum heat is greatest in the long wave-lengths. The explanation of the curious fact that this particular quality of heat may be more easily recognized where it exists in a less degree as in the lunar spectrum than where it is found in a relatively great degree as in the solar, will be still clearer if we consent (in continuance of the illustration) to further compare this lunar invisible radiation of great wave-length to the deep-red light from a piece of scarcely luminous hot iron. This peculiarly deep red is seen with little difficulty in the iron in a dark room, but never in daylight; yet it is of a quality which we know from theory must exist in far greater degree in the daylight itself; nor do we, even when we would isolate it in a certain part of the solar spectrum, see it there, because it is now obscured by the inevitable diffusion or reflexion of part of the neighbouring brilliant light which the prism ought to keep wholly away, but (owing to inevitable instrumental defects) does not. The dull glowing iron carries no white light along with it, and therefore its feeble peculiarly deep red is easier seen than the far stronger corresponding red in the solar spectrum.

By the aid of this analogy in the case of light, and passing now to the actual case of wholly invisible radiation, I hope it may be clear how the feebler heat in the lunar extreme infrared spectrum was at first recognized more easily than the stronger corresponding heat in that of the sun.

It may be asked why (if we cannot cut off the diffused heat in the solar infra-red spectrum by the use of an absorbing glass) we cannot put a prism in front of the slit after the plan of Helmholtz. This is practically impossible here (owing to instrumental conditions which we need not now explain), unless we find some way of keeping the axis of the spectrobolometer either motionless or always parallel to itself, in spite of the varying direction of the rays from such a prism, and of automatically limiting the kind of radiation to be observed in any part of the spectrum, to that legitimately belonging there. The following arrangement was, after various trials, adopted with success. Its immediate purpose is to overcome the difficulty which we have just explained at such length-that is, to sift out the extraneous heat which remains after the ordinary action of the prism; but it can of course be used for light also.

\section{Description of Sifting Train (see fig. 1).}

Let NS be a massive beam, resting on two piers, and immovably fixed in the meridian. Let $A R$ be a second beam, movable on a turn-table, placed centrally beneath N S. 


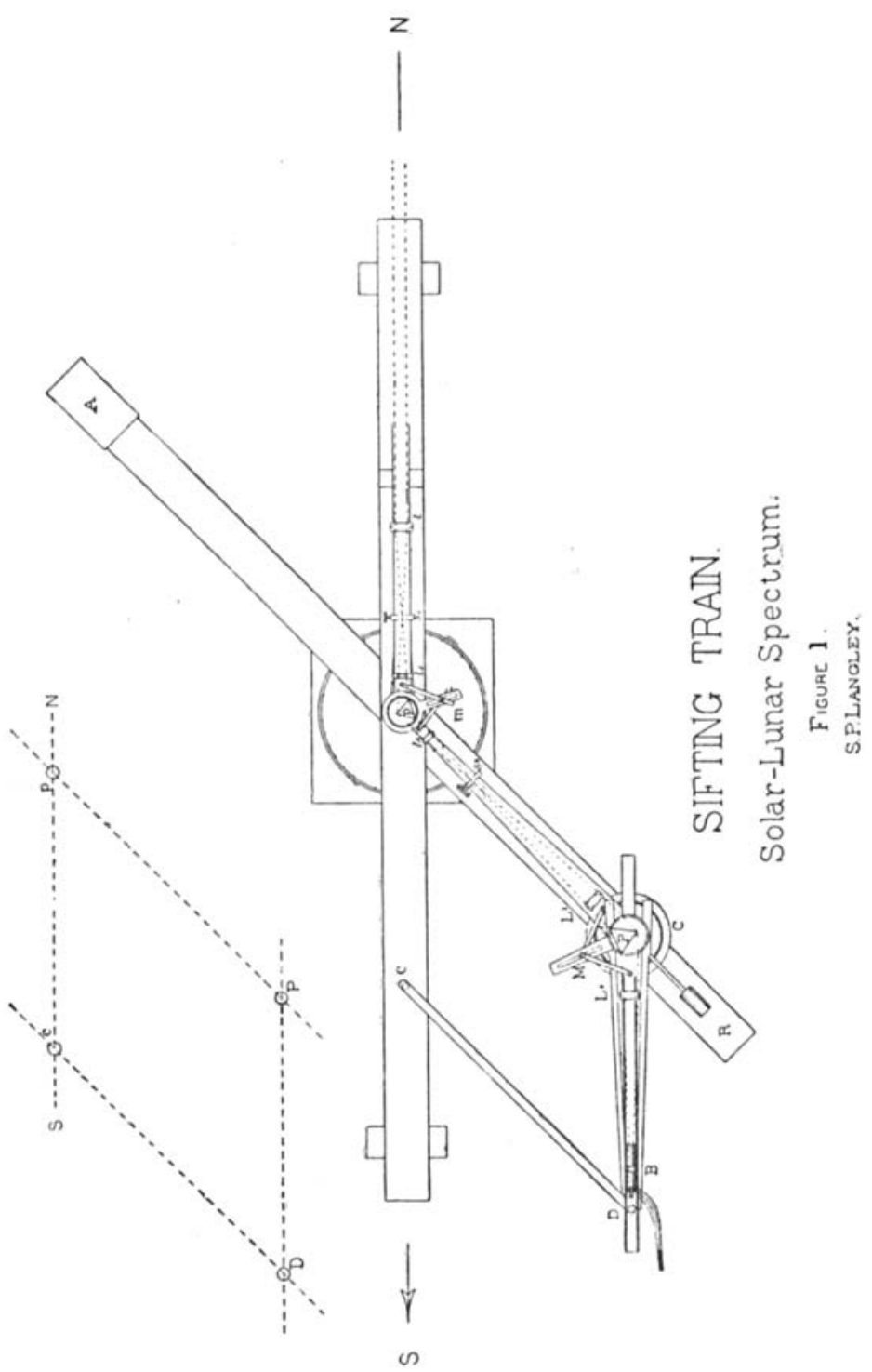


Temporarily mounted on $A R$, and moving about with it, is the large spectro-bolometer described in a previous memoir. The centre of its graduated circle (C) lies under the point P. Its two long arms are not free to move as usual, but are constrained by mechanical attachments (not here shown) to occupy the positions $\mathrm{P} p, \mathrm{PD}$.

Two large $60^{\circ}$ prisms of the same material (pure rock-salt from the same mine), their faces worked with the greatest accuracy, are placed with their equal refracting angles in opposite directions, one $(\mathrm{P})$ at the obtuse, the other $(p)$ at the acute angle of the parallelogram $(\mathrm{P} p \mathrm{D} c)$, the vertices of all whose angles in the mechanical construction are pivoted and connected by inflexible arms, so that (both prisms being kept automatically in minimum deviation by the attachments, $\mathrm{M} m$ ) the angle of minimum deviation $(c p \mathrm{P})$ for the first prism is necessarily equal to the angle of minimum deviation ( $R$ P D, or its equal $\mathrm{PD} c$ ).

Thus, if the pencil of solar lunar heat (reflected from a large siderostat on the north, not shown here) passes from $\mathrm{N}$ towards $\mathrm{S}$, on moving the beam $A \mathrm{R}$, pivoted at $p$ ( $p$ being the projection in our drawing of a vertical line passing through the centre of the turn-table and the median line of N S and A R) into various positions (N S remaining fixed), the rays, which are refracted by the prism $p$ in the direction $p \mathrm{P}$, will emerge from $\mathrm{P}$ in the direction $\mathrm{PD}$ and fall upon the bolometer B. A condensing-lens $(l)$ forms the solar image on the slit $\left(s_{1}\right)$ of the first spectroscope, whose train (consisting of collimator, $l_{1}$, prism, $p$, and image-forming lens, $l_{2}$ ) forms a spectrum on the slit $\left(s_{2}\right)$ of the second spectroscope. Here a narrow pencil from the first spectrum, comprising only the particnlar wave-lengths which fall within the width of $s_{2}$, is admitted, and, by the second train $L_{1} P L_{2}$, formed into a horizontal spectrum at and on either side of $\mathrm{B}$. When we move AR this spectrum moves in turn past the vertical linear thread of the bolometer $B$, which lies in the focal plane of this spectrum, and is immersed in its successive absorption-lines as these defile past it. The function of the first spectroscopic train $\left(l, l_{1}, p, l_{2}\right)$ is solely to sift out the extraneous radiations, and to present at the second slit $\left(s_{2}\right)$ only those which legitimately belong to that part of the spectrum we wish to study. These pure rays pass into the second slit and are analysed by the second train in the usual way, by the aid of the linear bolometer at B, and of the circle (C) reading to ten seconds of arc.

The objection to this apparatus is its complexity, which, however, we have been unable to advantageously diminish. 
We may, however, satisfy ourselves by visual observation of the Fraunhofer-lines seen through the whole compound system (entirely of salt) both of the optical perfection of the surfaces of our entire double train, and of the accuracy of its purely automatic action.

\section{Results of Observation.}

With this and the apparatus alreudy described in previous memoirs we have searched the extreme infra-red solar spectrum, at first without definite success, later with results which will be better understood by tho accompanying drawings. Fig. $2 b$ shows the nowly inrestigated invisible solar-heat spectrum on the normal scale up to an (estimated) wavelength of $18^{\mu}$. Fig. $3 a$ is an enlarged riew of that portion of it extending to $5^{\mu}$, and fig. $3 b$ a photographic interpretation of the last, obtained automatically by a special clevice; so as to present somewhat the appearance which this beat-region might be expected to show to an eye which could see it.

Inordinately long as our new chart (fig. 2U) may seem, we see that the scale is, nevertheless, contracted to the list degree, so that the entire visible spectrum is compressed into hardly an inch, seen on the left or violet end. Next we have tho already described solar infra-red, already shown to exist to $2^{\mu} \cdot 8$, and which includes its great absorption-bands $\Phi, \Psi, \Omega$, already investigated with glass prisms.

The principal lunar heat lies chiefly beyond the great wavelength of $0.01 \mathrm{millim} .\left(10^{\mu}\right)$, and ere we reach it we pass over a region between $5^{\mu}$ and $11^{\mu}$ (many times the length of the entire visible spectrum), where the solar heat seems to have been, to our present means of recognition, entiroly absorbed, probably chiefly by our own atmosphere.

It will be convenient, however, after noting the extent of the whole region shown in fig. $2 a$, to commence our deseription with the detailed portion shown in figs. $3 a$ and $3 l$, to which the reader is referred in illustration of what immediately follows.

The lowest bands already described, as seen in the spectrum of a flint-glass prism, are the small ones $\omega_{1}$ and $\omega_{2}$ near $2^{\mu}$. Below this point all is believed to be here given for the first time. The bands in this new region are undoubtedly due chiefly, if not wholly, to telluric absorption; and they are notably variable, depending on the season of the year, and still more on the hour of the day. As the sun sinks, its rays, passing through increasing air-masses, suffer absorptions which singularly change the appearance of the bands, as is shown in tigs. 4 and 5, which, however, are drawn upon the 
512
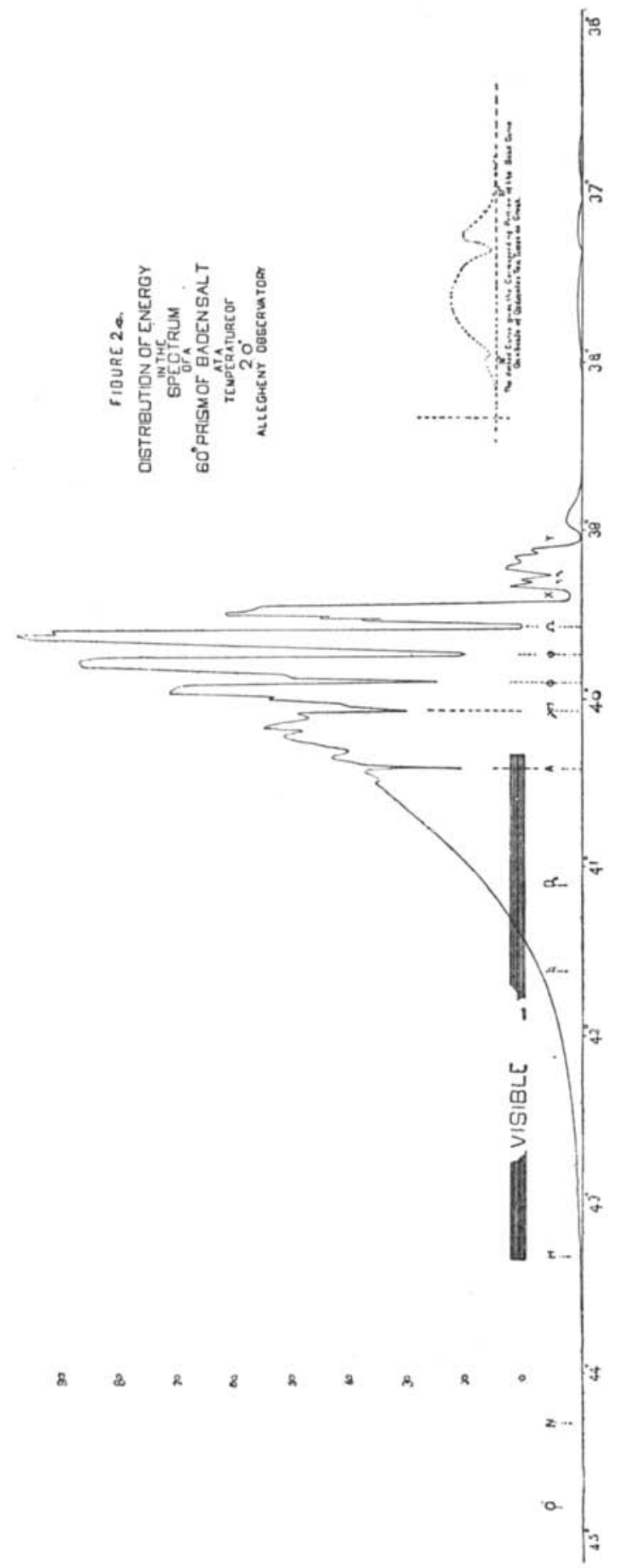


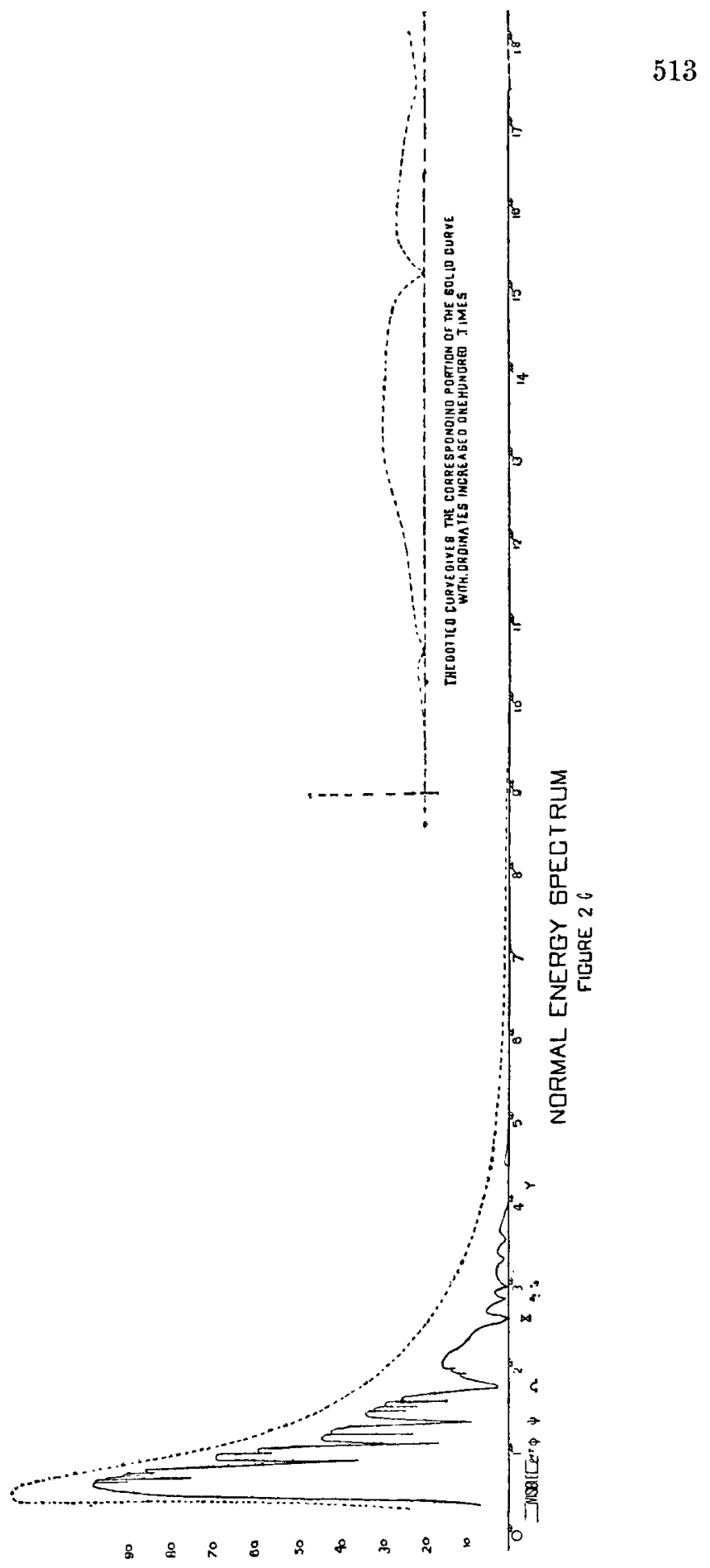


prismatic, not the normal, scale. They are identifiable with the portion extending from $\mathrm{X}$ to $\mathrm{Y}$ on the latter.

Observations made during the winter indicate that the band at $2^{\mu} \cdot 64$ (figs. $3 a, 3 b$ ) is, with a high sun, largely filled up, especially on the less refrangible side. At noon a subordinate maximum has been found within the low sun limits of this band at $2^{\mu} \cdot 94$, and a second one at $2^{\mu} \cdot 80$ frequently accompanies it, producing subordinate minima at $2^{\mu} \cdot 89$ and $3^{\mu} .02$.

As the absorption increases, with a sinking sun, these subordinate maxima disappear to a very great extent, that at $2^{\mu .} 80$ being the first to vanish as well as the quickest to grow, so that at noon, on a cold day, it not only surpasses the maximum at $2^{\mu} \cdot 94$, but even begins to approach that at $3^{\mu} \cdot 20$, while, when the sun's altitude is less than $10^{\circ}$, the nearly uniform part of the band extends from $2^{\mu} \cdot 45$ to $3^{\mu} \cdot 15$ without a break. Minor cold bands at $3^{\mu} \cdot 37$ and $3^{\mu} \cdot 69$ are suspected. The evidence for their existence may be seen by inspecting the high sun and low sun curves given in fig. 4 . From $4^{\mu} \cdot 0$ to $4^{\mu} \cdot 5$ we have another region of almost complete absorption, followed by a maximum at $4^{\mu} \cdot 6$, beyond which lies the longest break of all, stretching from $5^{\mu}$ to $11^{\mu}$.

The solar heat throughout the region from $5^{\mu}$ to $11^{\mu}$ is probably far greater than it here appears, and if the parts struck out by our atmosphere were restored it would probably be found that a not wholly inconsiderable portion of the sun's heat lies in this region; for it must be borne in mind that even the maxima are doubtless in some degree affected by a linear absorption, so that, because a part of the spectrum appears to be almost entirely transmitted, we cannot infer that it necessarily is so, or that it has not, after passing through the upper strata of the air, already parted with a considerable portion of its energy.

While the position of a line or band caused by light from the centre of the sun is, in the upper spectrum, unchangeable, in this extreme low spectrum (if we could photograph it, Jike the upper, on cold and on hot days, with high sun and with low) the absorption would be seen to increase not symmetrically with the centre of the band, but more on one side than the other, so as to considerably modify the position of greatest absorption. This seems to be the explanation of a curious fact which could not have been anticipated in advance of observation ; that is, that the centres of several of these bands and lines are under some conditions found to be shifted to a recognizable extent, and hence their wave-lengths are, within certain limits, variable.

It seems as though the absorption, which we see exercised 


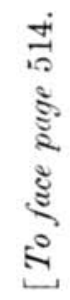

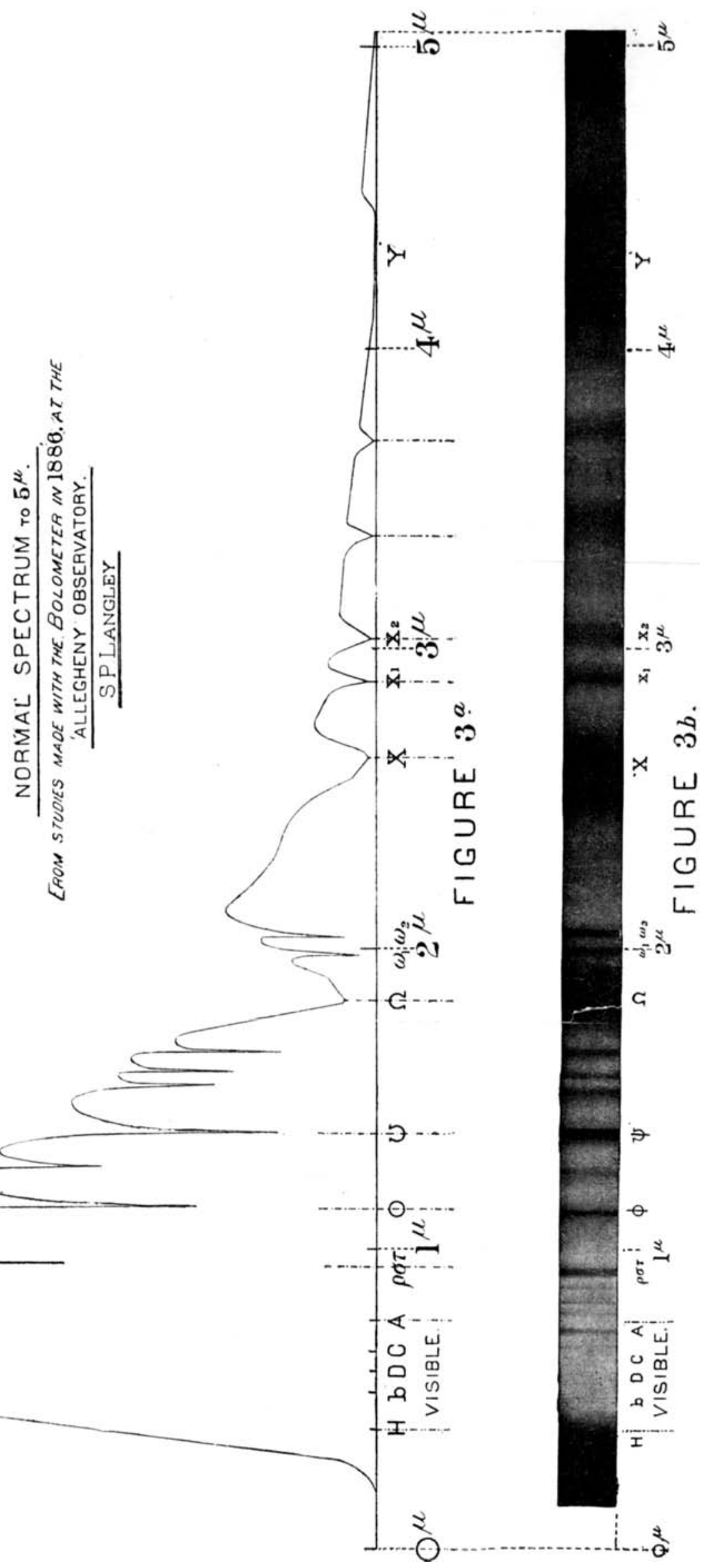


Solar and Lunar Spectrum.
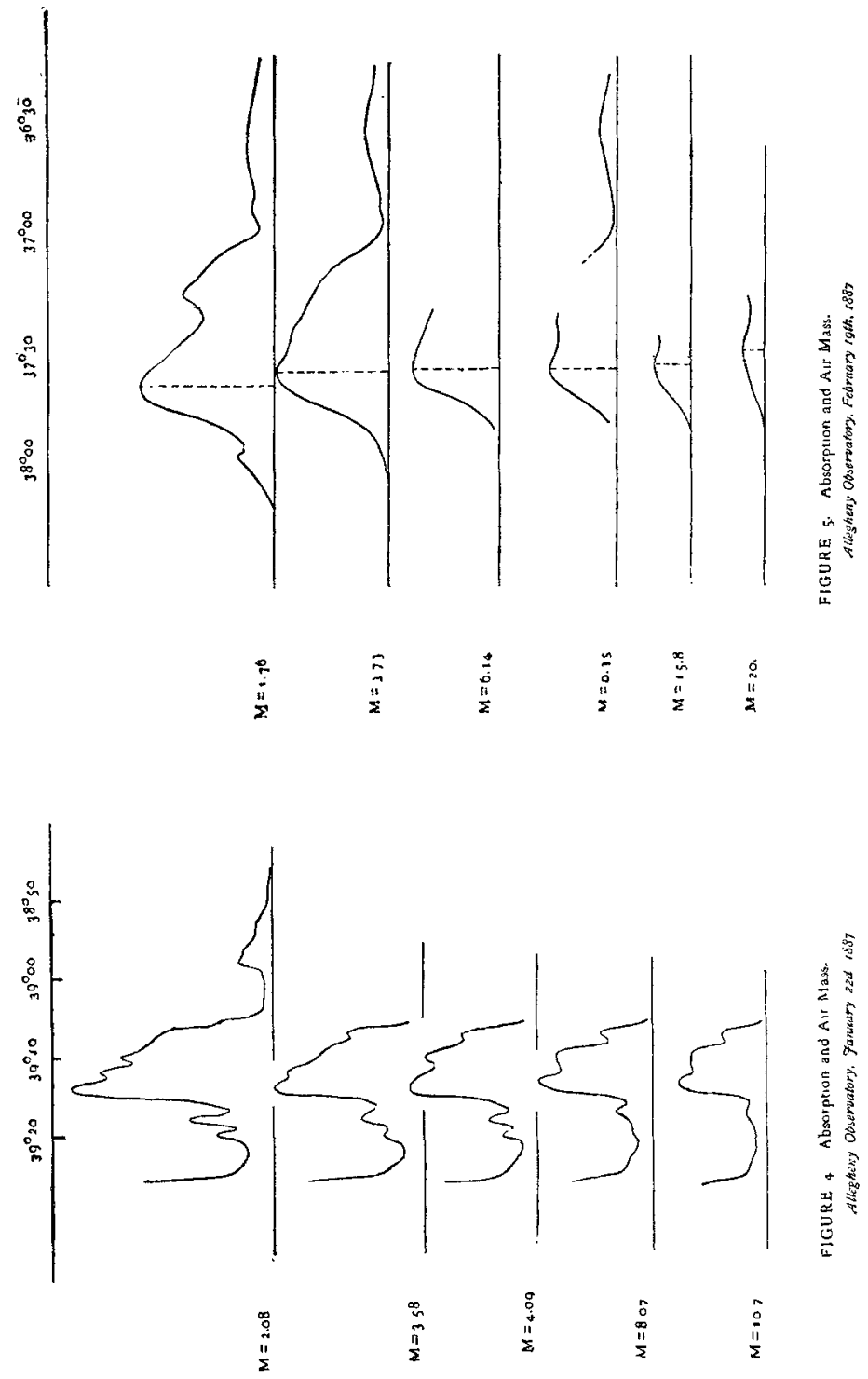
in most of the visible spectrum by fine lines, which begin already to show aggregation into a broader absorption-band on the borders of the infra-red (e.g. the well-known "A" of Fraunhofer), is, as we pass further down into the invisible part, represented by wider and wider bands, like $\Phi, \Psi, \Omega$ (composed themselves probably of lines). The heat between these local regions of almost total absorption continues, as I have repeatedly before stated, to be (contrary to the old belief) apparently transmitted with even greater facility than that in the visible spectrum. These bands have grown larger and closer and closer together as we have come down from the visible spectrum below the point where the old map ended, and not far below $5^{\mu}$ they seem to practically coalesce into one almost unlimited cold band. We do not, let it be observed, assert that the absorption is absolutely total even here, and, in fact, there is always a feeble heat to be observed throughout this extent. This, however, the use of the sifting train shows to be largely, at any rate, factitious, but we admit the possibility that subsequent research may prove that it is not all so.

Let us now recur to fig. $2 b$, where we shall find below $10^{\mu}$ the same dependence of the effects upon the season and the hour, as in the part above $5^{\mu}$.

At $10^{\mu \cdot 2}$ observations made during the autumn showed scarcely the feeblest suspicions of heat, and the same has held good in the very mild weather of the past winter (of 1887); but on a few days, when the temperature had fallen below the freezing-point, a notable maximum was found at this point, followed by a minimum at $10^{\mu \cdot 7}$. The height of this maximum relatively to the principal one in this region at about $13^{\mu}$ appears to be correlated with the composition of the air as affected by the temperature. On the coldest day (temperature at noon $-6^{\circ} .7$ C.) the deflection at midday for $\lambda=10^{\mu} \cdot 2$ was nearly one-half that at $13^{\mu}$; but on other days, when the temperature was near $0^{\circ} \mathrm{C}$., the deflection at $10^{\mu} \cdot 2$ did not exceed onefourth that at $13^{\mu}$, while at temperatures above $+10^{\circ} \mathrm{C}$. it was not noticeable.

It is in the region near $13^{\mu}$ to $14^{\mu}$, or over twenty times the length of the visible spectrum below it, that we have found the maximum of the lunar heat-spectrum; and it is here that we first obtained indications of solar radiation corresponding in its great wave-length to this special lunar radiation, but of amounts nonestimable by the means till now employed. I have already spoken of its almost unrecognizably small amount, and a perhaps more vivid apprehension of its extreme minuteness will be gained from the statement that on this graphical construction, on the scale of ordinates used in delineating the curve from 
0 to $5^{\mu}$, no heat appears below $5^{\mu}$ anywhere, not even at the maximum near $13^{\mu}$ and $14^{\mu}$; because, though heat exists to the bolometer, the highest ordinate which would represent it on our drawing is not so great as the thickness of the thin black line, which denotes the axis of abscissæ. I have accordingly here been obliged to exhibit it separately by a dotted curve whose ordinates are one hundred times those in the remainder of the spectrum. The points of minima in it are identifiable with absorption-bands, which we have directly observed and which we have independently found to exist in our own atmosphere by studying the radiations from a copper surface, one metre square, at the temperature of boiling water placed in the open air at a distance of one hundred metres from the bolometer. (The description of this will be found in a supplementary research to be given elsewhere.) The principal lunar heat, then, is found here, at a point of the spectrum corresponding to the maximum radiations from melting ice, but its maximum amount is probably less than 1 per cent. of the corresponding solar heat which we have just found to be itself so small. That we can detect the lunar heat at all under these circumstances is due to the fact that we are bere able to employ for it very short-focused mirrors and lenses, which condense it into a very short and relatively hot spectrum (there being no fear of their diffusing extraneous heat, since none worth mention exists). In the case of the sun we must employ a wholly different optical train, forming a far longer spectrum. It would be easily understood that these means, which enable us to determine the position of the solar and lunar heat-maxima here, are not favourable to a determination of the relative amounts of heat received from the sun and moon under such different conditions. We can only say that these ratios are themselves utterly changed from what they are in the visible spectrum, where we all know that the solar light is something like five hundred thousand times moonlight. It is probable that the solar heat received in this part of the spectrum is less than five hundred times the lunar; but the actual ratio is only very roughly determinable by our present means.

By comparison with the "heat"-spectra given in a previous memoir, we may also note the fact that some of the wavelengths given from ice are identifiable in the solar spectrum, nor (in view of the now established facts that the ratios of the heat at different parts of the spectra of two unequally hot bodies are functions of the wave-length) need it surprise us that we bave also found that this part of the spectrum of the sun is not incomparably hotter than the corresponding part of the ice-spectrum.

Phil. Mag. S. 5. Vol. 26. No. 163. Dee. 1888. $2 \mathrm{~N}$ 
It may be asked if we can, after all, feel sure of the character of such minute amounts of heat in the presence of the already described reflected and diffused heat from the upper spectrum, considering the possibility that something may go wrong in the elaborate arrangement of the sifting-train, and leave us (as everything we study now is invisible) without ocular warning of the fact. I may reply that we have lately found an admirable check upon the efficiency of our optical devices in the behaviour of that familiar substance lampblack, which all physicists use either on thermometers, thermopiles, or bolometers. All of us know probably that it is not absolutely non-selective, as it used to be thought, and that it has a tendency to transmit the infra-red with greater freedom than the visible spectrum; but the statement I am about to make may excite surprise. It is that when a very perfectly polished. rock-salt plate is covered by a sheet of lampblack of such thickness as to transmit less than one per cent. of ordinary white light, it transmits about ninety per cent. of the radiations belonging to these extreme wave-lengths. In other words, it has become a transparent body to rays of this wave-length, while it exercises an intermediate degree of absorption on intermediate rays, so that by the amount of their absorption by lampblack we have a test by which these latter may be independently identified.

We have given this study not only to map new lines and bands in the region between $3^{\mu}$ and $5^{\mu}$, but also to verify the existence of an all but infinitesimal amount of heat at much greater wave-lengths. We have applied great pains to this latter, not so much on account of its own importance as on account of the important conclusions to be drawn from it later; for if it is true that in this extreme spectral region, corresponding to temperatures much below the boiling-point and even below that of melting ice, the amount of the solar heat is trivial, it is also true that the fact of its existing at all is of very significant interest to the meteorologist ; for it is obviously here, at temperatures below $100^{\circ} \mathrm{C}$., that the rays which make up the nocturnal as well as diurnal radiations from the soil of our own planet are to be found. We observe that if such rays can enter the air from the sun, they can go out even from an icy soil (and still more from an ordinary one), to whose radiations it hence appears the atmosphere is more or less permeable. Heat, then, apparently escapes in some very minute degree even from the surface of the Arctic regions, not only by convection but by direct radiation through the atmosphere towards space. Meteorological questions of great interest, to which we shall return in a later 
memoir, can best be answered from a study of this region; and even if the problem of the lnnar temperature did not interest us as students of celestial physics, we should still find others depending on it of very practical import.

As to the degree of accuracy obtainable in fixing its position, we may remind the reader that the old formulæ of Canchy and others being useless, as we have shown, we have already undertaken a research * to enable us to determine such wavelengths in this region. We have determined all the wavelengths by the interpolating curve made from the observations of this year, which has been exhibited to the Academy at its April meeting. In this memoir will be found all the explanations we can offer relating to the degree of trustworthiness of the values assigned to the present extreme wave-lengths. If they are, as we believe, to be trusted within the limits there given, we have measured indications of lunar heat (and possibly of solar) greater than are shown on this drawing, and whose wave-lengths exceed one fiftieth of a millimetre. It is not likely that the more refrangible of that extremely feeble heat, which we here particularly describe, is much less than $11^{\mu}$.

I think we may now feel justified in saying that we probably know some of the main facts about the solar spectrum, so far as terrestrial absorption is concerned. Broadly speaking, they are these :-

Hardly one fourth of the solar energy, as we get it, is visible, at least without special precautions. Of the remaining three fourths, by far the larger portion of the heat actually received lies in the region above $2^{\mu} \cdot 8$, which has already been delineated, but if it were not for terrestrial absorption, the heat in the region below it might not improbably be relatively greater.

The effects of terrestrial absorption appear in the visible spectrum chiefly by means of the Fraunhofer lines, so that our first impression on looking at it is that these lines only occasionally interrupt the play of light and colour by which the solar energy makes itself known through the sense of vision. As we go down into those lower parts of the infra-red region, we find (directly contrary to the old belief) that, broadly speaking, the heat apparently grows more and more transmissible by our atmosphere, and this because the heat between the lines grows more and more transmissible, while the lines themselves, though growing into broader bands of almost total absorption, have not yet extinguished the hot regions

* "On hitherto unrecognized Wave-lengths," Phil. Mag. August 1886. 
between them, so that, even taking regions of transmission and absorption together, on the whole here, i.e. above $3^{\mu}$, more of dark heat in proportion is, perhaps, transmitted than of light heat.

We may roughly illustrate* this portion of the sun's spectrum after absorption by saying that, if it were visible, it would present almost the appearance of diffuse luminous bands on a dark field, somewhat like those seen in the spectra of stars of the fourth type. As we have observed that absorption seems to increase in the same direction in the sun's own atmosphere as in ours, it would be interesting to know if any thing analogous exists in the absolute solar spectrum, i.e. before absorption in the sun's atmosphere, but this we are not as yet able to determine.

Since, in the part of the spectrum described here for the first time, the same process of aggregation into ever wider and wider regions of absorption is continued till these intermediate regions of transmission disappear, the whole, to repeat an expression I have used in a earlier memoir, seems to become one continuous cold band, in which, however, we have found a little heat struggling through in the part beyond $11^{\mu}$. Briefly, then, we may say, that to an eye which could see the whole spectrum, visible and invisible, the luminous part being, as we know, interrupted by occasional dark lines, the lower part to $5^{\mu}$ would appear to consist of alternate bright and dark bands, and the part below $5^{\mu}$ be nearly dark, but with feeble "bright" bands at intervals.

In conclusion, we may say that these new researches extend the known solar spectrum from three to much over eighteen microns, shown on our plate, and while confirming the previously announced fact that the solar heat which reaches us here is negligible in amount, show from the fact of the existence of any at all, that the anomaly of our being able to perceive lunar heat where we could not formerly detect solar, can be explained consistently with the possible existence in the latter of every wave-length before absorption.

These investigations into a problem of Solar Physics have also incidentally led us to the prospective means of solution of an important one in Meteorology, for they have opened to observation the hitherto unknown region of the spectrum in which the nocturnal and diurnal radiations, not only from the moon toward the earth, but from the soil of the earth toward space, are to be found and may be hereafter studied in detail.

* Figure 3. 